\title{
Neonatal screening, clinical features and genetic testing for galactosemia
}

To the Editor:

Neonatal screening has been introduced in many countries in order to detect neonates affected by metabolic or endocrine diseases when they are still without symptoms. The neonatal screening for metabolic diseases may include galactosemia. The hallmarks of classical galactosemia [galactose-1-phosphate uridyltransferase (GALT) deficiency] are Gram-negative sepsis, early acute hepatic dysfunction, and failure to thrive. These neonates are already symptomatic before neonatal screening results will be obtained. To diagnose most of the 
newborns with severe symptoms, improved clinical vigilance and selective screening for galactosemia have been proposed. ${ }^{1}$

Neonatal screening for galactosemia has been in effect in Northeast Italy for many decades. During the last 3 years, we screened about 186,000 neonates and detected three cases of GALT deficiency and one case of galactokinase (GALK) deficiency.

The first case (7 days old) showed sepsis-like symptoms, vomiting, severe loss of body weight, and liver and kidney dysfunction. The clinical diagnosis of GALT deficiency was confirmed by laboratory tests before the neonatal screening results were obtained. Genetic investigation of GALT gene revealed a compound heterozygosity for a Q188R and a M142T mutation. Q188R (Gln-Arg exchange in exon 6) is the most frequent mutation in European populations. ${ }^{2}$ M142T (Met-Thr exchange in exon 5) is a novel mutation occurring in a conserved region, considered to be a hot spot for mutations (G. Novelli, unpublished data, 2004).

The second case ( 6 days old) showed sepsis-like symptoms, hepatitis, coagulopathy, vomiting, icterus, and encephalopathy. An emergency evaluation confirmed GALT deficiency. Residual GALT activity in erythrocytes was $<0.1 \mu \mathrm{mol} /$ $\left(\mathrm{h}^{\star} \mathrm{g}_{\mathrm{Hb}}\right)$. Genotyping revealed a compound heterozygosity for the most common Q188R and the rare Y286C mutation (Tyr-Cys exchange in exon 9).

Recently, another case of GALT deficiency was diagnosed. He showed after birth low feeding tolerance, liver dysfunction, anemia, and echo-tomography signs of brain injury. Initially, he was inappropriately diagnosed having sepsis. GALT activity was $0.3 \mu \mathrm{mol} /\left(\mathrm{h}^{\star} \mathrm{g}_{\mathrm{Hb}}\right)$. Mutation analysis showed compound heterozygosity for R333W (Arg-Trp exchange in exon 10) and T6fsdelC, a small deletion causing frame shift in exon 1 (P. Schadewaldt, unpublished data, 2004).

Neonatal screening for galactosemia can also detect a GALK deficiency. This was the case of a female (17 days old) who had a very low GALK activity. An ophthalmologic examination showed a cataract in the right eye and a light opacity in the left eye; both regressed with galactose-free diet. Family was of Bosnian descended. Both parents and the 4 -year-old sister were heterozygous carriers. Surprisingly, an older 9-year-old sister with GALK activity close to zero had a normal ophthalmologic evaluation. Molecular analysis showed the presence of the heterozygous P28T mutation, most likely of Bosnian ancestry. ${ }^{3}$

Due to the early onset of symptoms, a screening program for galactosemia can confirm only the clinical suspicion of GALT deficiency. Rare cases of these neonates not appropriately diagnosed after birth may be correctly diagnosed with a neonatal screening program.

In the unscreened population, asymptomatic patients with GALK deficiency may be diagnosed later than in the screened ones. However, this measure allows to prevent the worsening of ophthalmologic damage.

To obtain as rapidly as possible the results of the neonatal screening, the samples should be mailed by express mail (or by courier), and the work of the screening laboratory should not suffer from festive standstills. Obviously, the results would be better if newborn screening took place at day 3 using combined substrate screening and enzymatic testing. These procedures may enhance the costs of the neonatal screening without an impact on the long-term complications. ${ }^{4}$ In all neonates with sepsis and liver disease, an index of suspicion of galactosemia (GALT deficiency) must be done; also, if there is a neonatal screening program and institution of lactose free diet in a very early stage is essential. In these selected cases, the blood samples may be sent by express mail. Instead, because clinically asymptomatic, only the patients with GALK deficiency always benefit from the screening program.

\section{ACKNOWLEDGMENTS}

Work in part supported by a grant from the Italian Ministry of Health to G.N.

Marco Zaffanello, MD

Giorgio Zamboni, MD

Department of Paediatrics

University of Verona

Verona, Italy

Peter Schadewaldt, MD

Klinik fur Allgemeine Padiatrie

Universitatsklinikum, Heinrich-Heine-Universitat Dusseldorf

Dusseldorf, Germany

Paola Borgiani, MD

Giuseppe Novelli, MD

Department of Biopathology and Diagnostic Imaging

University of Roma Tor Vergata

Rome, Italy

\section{References}

1. Shah V, Friedman S, Moore AM, Platt BA, Feigenbaum ASJ. Selective screening for galactosemia: an alternative approach. Acta Paediatr 2001;90:948-949.

2. Tyfield L, Reichardt J, Fridovich-Keil J, Croke DT, Elsas LJ II, Strobl W et al. Classical galactosemia and mutations at the galactose-1-phosphate uridyl transferase (GALT) gene. Hum Mutat 1999;13:417-430.

3. Reich S, Hennermann J, Vetter B, Neumann LM, Shin YS, Soling A et al. An unexpectedly high frequency of hypergalactosemia in an immigrant Bosnian population revealed by newborn screening. Pediatr Res 2002;51:598-601.

4. Schweitzer-Krantz S. Early diagnosis of inherited metabolic disorders towards improving outcome: the controversial issue of galactosaemia. Eur J Pediatr 2003;162:S50-S53. 